\title{
APPLICATIONS OF GAUSS'S PRINCIPLE OF LEAST CONSTRAINT TO THE NONLINEAR HEAT-TRANSFER PROBLEM
}

\author{
B. VUJANOVIC and B. BACLIĆ \\ Faculty of Technical Sciences, University of Novi Sad, 21000 Novi Sad, Yugoslavia
}

\begin{abstract}
An approximate direct method for solving linear and nonlinear heat conduction problems, based on the Gauss's principle of least constraint is presented. In every particular case, the problem is reduced to the algebraic minimization of a quadratic form with respect to some complex of physical parameters. By the help of several concrete examples the efficiency and accuracy of this new method is demonstrated.
\end{abstract}

\section{NOMENCLATURE}

a, acceleration;

b, characteristic length;

c, specific heat;

$E$, percentage error;

F, force;

Fo, Fourier number $\alpha t / l^{2}$;

$H$, coefficient;

$h$, convective heat-transfer coefficient;

$K$, complex representing the component of spatial change of temperature distribution;

$k$, thermal conductivity;

$L$, latent heat of melting;

$l$, characteristic length;

m, mass;

$q$, penetration depth;

r, position vector;

$t$, time;

$T$, nondimensional temperature;

v, velocity;

$W, \quad$ complex representing the component of temporal change of temperature distribution;

$x$, length coordinate;

$X$, nondimensional length $x / l$;

spatial change of temperature distribution (10);

$y$, length coordinate;

$Y$, nondimensional length $y / b$; temporal change of temperature distribution (10);

Z, Gauss's constraint.

Greek symbols

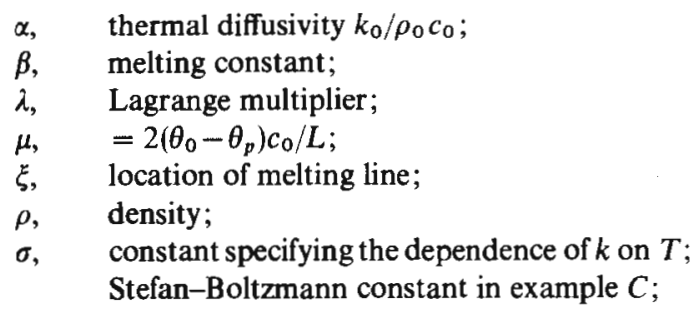

\author{
$\theta$, temperature; \\ $\theta_{p}, \quad$ temperature of phase change.
}

Subscripts

0 , refers to initial values;

$f$, refers to the fluid;

$m$, refers to the surrounding medium;

$S$, refers to the surface of the body.

\section{INTRODUCTION}

THE USE of variational methods for obtaining solutions of transport phenomena has become an indispensable tool in the domain of nonlinear conduction. Many publications dealing with variational principles of various kinds have appeared in the technical literature in the past 20 years. Roughly speaking, all variational principles in use at the present time in the study of heat transfer, are built up in formal analogy with known principles of classical mechanics. This fact confirms the claim of Heisenberg that: "Mechanics could be a model and a basis for all other fields of science ..." ([1], p. 89). The most popular principles of mechanics which are advantageously used in many branches of engineering as a starting point for the direct calculation are the D'Alambert's principle of virtual work and Hamilton's principle of stationary action. In addition, when a physical problem is stated in the form of Hamilton's principle it becomes a variational problem in the sense of variational calculus. The direct methods applied on the D'Alambert's principle are generally known as the Galerkin's method, while the numerous direct computations based on the Hamilton's principle are known as Ritz's method, method of partial integration, Rayleigh's method etc.

In this paper we shall study the possibility of applications of Gauss's principle of least constraint to the nonlinear heat transfer. This principle is a true minimum principle in contrast with the two aforementioned principles of D'Alambert and Hamilton, which are generally not the minimum principles.* Contrary to

*The variational principle of Hamilton may be occasionally a true minimum principle. 
the general impression that the Gauss's principle is of purely theoretical interest in classical (point) mechanics, the purpose of this, and the previous article [2] is, to point out that this principle can lead to considerable practical advantages in nonlinear heat transfer.

\section{GAUSS'S PRINCIPLE}

For the sake of clarity we will briefly describe the Gauss principle of ordinary mechanics in a form suitable for applications in heat transfer.

Consider a free dynamical system of $n$ particles, subject to impressed forces $\mathbf{F}_{i}(i=1,2, \ldots, n)$. If $m_{i}$ are masses, $\mathbf{r}_{i}$ position vectors and $\mathbf{a}_{i}=\mathrm{d}^{2} \mathbf{r}_{i} / \mathrm{d} t^{2}$ accelerations, the differential equations of motion are

$$
m_{i} \mathbf{a}_{i}-\mathbf{F}_{i}=0
$$

Let us introduce the quantity*

$$
Z=\frac{1}{2} \sum_{i=1}^{n}\left(\mathbf{F}_{i}-m_{i} \mathbf{a}_{i}\right)^{2}
$$

and suppose that the configuration $\left(\mathbf{r}_{i}\right)$, velocity $\left(\mathbf{v}_{i}\right)$ and forces $\left(\mathbf{F}_{i}\right)$ of the system are prescribed at time $t$, i.e.

$$
\delta \mathbf{r}_{i}=0, \quad \delta \mathbf{v}_{i}=0, \quad \delta \mathbf{F}_{i}=0
$$

and the remaining "complex"- $m_{i} \mathbf{a}_{i}$ is then the only one to be varied, i.e.

$$
\delta\left(m_{i} \mathbf{a}_{i}\right) \neq 0 .
$$

The Gauss's principle of least constraint states that under the conditions (3) and (4) the quantity $Z$ can assume its absolute minimum, which is zero. The proof is simple. If $\left(-m_{i} \mathbf{a}_{i}\right)$ represents the actual inertial force, and $-m_{i} \mathbf{a}_{i}+\delta\left(m_{i} \mathbf{a}_{i}\right)$ represents any other possible inertial force, we have:

$$
\begin{aligned}
\delta Z & =\frac{1}{2} \sum_{i=1}^{n}\left\{\left[\mathbf{F}_{i}-m_{i} \mathbf{a}_{i}+\delta\left(m_{i} \mathbf{a}_{i}\right)\right]^{2}-\left(\mathbf{F}_{i}-m_{i} \mathbf{a}_{i}\right)^{2}\right\} \\
& =\frac{1}{2} \sum_{i=1}^{n}\left[\delta\left(m_{i} \mathbf{a}_{i}\right)\right]^{2}+\sum_{i=1}^{n}\left(\mathbf{F}_{i}-m_{i} \mathbf{a}_{i}\right) \delta\left(m_{i} \mathbf{a}_{i}\right)
\end{aligned}
$$

and the last term vanishes in virtue of (1). Thus

$$
\delta Z>0
$$

unless $\delta\left(m_{i} \mathbf{a}_{i}\right)$ is zero.

As a second possibility $\dagger$ of achieving the absolute minimum of "constraint" $Z$ defined by (2), we can suppose that the configuration, velocity and acceleration of the system are prescribed at time $t$ and the impressed force $F_{i}$ is then only one to be varied, i.e.

$$
\delta \mathbf{r}_{i}=0, \quad \delta \mathbf{v}_{i}=0, \quad \delta\left(m_{i} \mathbf{a}_{i}\right)=0, \quad \delta \mathbf{F}_{i} \neq 0 .
$$

If the system in question is subject to holonomic or nonholonomic constraints the constraint $Z$ is in minimum again but introduction of Lagrange's undetermined multipliers is necessary.

We will show now how one can treat the equations of nonlinear heat transfer in the same way as the dynamical systems discussed above.

* In the original version of Gauss's principle the expression in the parenthesis of (2) is multiplied by the factor $1 / m_{i}$.

†For more details see [2].
Consider the nonlinear equation of heat conduction

$$
\operatorname{div}(k \cdot \operatorname{grad} T)-\rho c \frac{\partial T}{\hat{\imath} t}=0 .
$$

where $\rho$ is the density, $k(T)$ and $c(T)$ are the thermophysical coefficients which are supposed to be the functions of temperature $T$.

Consider the heat-transfer analog of equation (2):

$$
Z=\int_{V}[X-Y]^{2} \mathrm{~d} V
$$

where $V$ is the volume which is engaged in the process of heat transfer and

$$
\begin{aligned}
& X \equiv \operatorname{div}(k \cdot \operatorname{grad} T) \\
& Y \equiv \rho c \frac{\partial T}{\partial t}
\end{aligned}
$$

are spatial and temporal parts respectively. As in the previous case the following two variational rules for minimizing (9) are possible

$$
\begin{array}{ll}
\delta X \neq 0, & \delta Y=0 \\
\delta X=0, & \delta Y \neq 0 .
\end{array}
$$

This variational principle may be formulated by means of the generalized coordinates instead of the components of the field itself. In numerous problems it is possible to guess that a solution belongs to a family of functions with one or more unknown parameters, which is more or less characteristic feature for all approximate methods. In each particular case we must be able to identify the characteristic complex of parameters which represent $X$ or $Y$ and minimization of $Z$ has to be performed with respect to one of these complexes. The method proposed is very simple because the whole technique for obtaining approximate solution is of the algebraic nature-minimization of a quadratic form with respect to a complex. If some of generalized coordinates are coupled by one or more algebraic relations the minimization of $Z$ should be performed using Lagrange's undetermined multipliers.

The efficiency of the method can be illustrated by obtaining approximate solution of a simple linear heat-transfer problem.

Consider a one-dimensional thermally insulated semi-infinite body with constant thermo-physical coefficients $\rho=\rho_{0}, c=c_{0}$ and $k=k_{0}$. The body is initially at the temperature $T=0$. At $t=0$ the face of the body, located at $x=0$, is suddenly brought to a constant temperature $T=T_{0}$. Mathematically the problem is to solve following boundary-value problem

$$
\frac{\partial T}{\partial t}=\alpha \frac{\partial^{2} T}{\partial x^{2}}
$$

where $\alpha=k_{0} / \rho_{0} c_{0}$, together with

$$
T(0, t)=T_{0} ; \quad T(x, 0)=0 .
$$

Following the concept of the penetration depth assume the solution in the form

$$
T=T_{0}\left(1-\frac{x}{q(t)}\right)^{2}
$$

where $q(t)$ is penetration depth. 
Let us solve the problem minimizing the constraint:

$$
Z=\int_{0}^{q}\left[\frac{\partial T}{\partial t}-\alpha \frac{\partial^{2} T}{\partial x^{2}}\right]^{2} \mathrm{~d} x
$$

with respect to the temporal part $\partial T / \partial t$. From (15) we have

$$
\begin{gathered}
Y=\frac{\partial T}{\partial t}=2 T_{0}\left(1-\frac{x}{q}\right) \frac{x}{q} \cdot W \\
\frac{\partial^{2} T}{\partial x^{2}}=\frac{2 T_{0}}{q^{2}}
\end{gathered}
$$

where

$$
W=\frac{\dot{q}}{q}
$$

is the "temporal complex" with respect to which we will minimize the expression (16). Substituting (17) into (16) integrating and retaining only the terms with $W$ we have

$$
Z(W)=\frac{W^{2} q}{10}-\frac{W \alpha}{q}
$$

where a constant multiplicative factor has been omitted. Minimizing (19) with respect to $W$, i.e.

$$
\frac{\partial Z(W)}{\partial W}=0
$$

and using (18) we find the following differential equation

$$
q \dot{q}=5 \alpha
$$

the solution of which, with respect to the initial condition $q(0)=0$ is

$$
q=\sqrt{ }(10 \alpha t)
$$

Finally let us use the second possibility, minimizing (16) with respect to the spatial complex

$$
X=\alpha \frac{\partial^{2} T}{\partial x^{2}}=2 K T_{0}
$$

where

Introducing

$$
K=\alpha / q^{2}
$$

$$
\frac{\partial T}{\partial t}=2 T_{0}\left(1-\frac{x}{q}\right) \frac{x}{q^{2}} \dot{q}
$$

and (21) into (16), retaining the terms with $K$, we have after integration

$$
Z(K)=K^{2} q-\frac{1}{3} K \dot{q}
$$

where a constant multiplicative factor has been omitted. The equation $\partial Z(K) / \partial K=0$, together with (22) will yield the equation

$$
\begin{gathered}
q \dot{q}=6 \alpha \\
\text { i.e. } \quad q=\sqrt{ }(12 \alpha t) .
\end{gathered}
$$

These two results (20) and (24) are in good agreement with the exact solution. Besides, they are identical with the approximate solutions obtained by the Galerkin and integral methods respectively.
It should be noted that the Gauss principle of least constraint has been applied on the linear heatconduction problems by Samoilovich in [3]. However, the author deals with a transformed form of the basic heat conduction equation similar with the Biot's quasivariational method.

It is the purpose of this note to show that the Gauss's principle can be applied directly on the governing heat-conduction equations, and in addition, the nonlinear problems are generally treated in the same way as the linear ones.

As was shown above, the applications of direct methods, using Gauss's principle are simple and straightforward. In the next section several more complex examples will be presented.

\section{EXAMPLES}

(A) Unsteady two-dimensional nonlinear heat conduction through the prism-like infinite bodies with a given cross section

As the first example we shall study the temperature distribution through the prism-like infinite bodies. The thermal conductivity is supposed to be a linear function of temperature hence the differential equation is of the form

$$
\frac{\partial T}{\partial t}-\alpha \frac{\partial}{\partial x}\left[(1+\sigma T) \frac{\partial T}{\partial x}\right]-\alpha \frac{\partial}{\partial y}\left[(1+\sigma T) \frac{\partial T}{\partial y}\right]=0
$$

where $\alpha$ and $\sigma$ are given constants.

Initially, the body is at the uniform temperature, i.e.

$$
T(0, x, y)=T_{0}=\text { const. }
$$

and the surfaces of the body are maintained at the zero temperature

$$
\left.T(t, x, y)\right|_{s}=0
$$

where $\left.\right|_{s}$ is the symbol for the external surfaces of the prism. Consequently the problem is to find the approximate solution of (25) in the presence of initial and boundary conditions (26) and (27).

The suitable form of the trial solution will be:

$$
T=\phi(t) B(x, y)
$$

where $B(x, y)$ is a specified function of the position which has to be chosen in accordance with the shape of the cross section of the body. The function $B(x, y)$ has the following properties: $\left.B(x, y)\right|_{s} \equiv 0$, and $B(x, y)>0$ for every $x$ and $y$ inside the region bounded by $S$. Hence, the problem is reduced on finding the unknown function of time $\phi(t)$, using the Gauss's principle.

Let us consider the constraint in the same sense of Gauss :

$$
\begin{array}{r}
Z=\int_{x_{0}}^{x_{1}} \int_{y_{0}}^{y_{1}}\left\{\frac{\partial T}{\partial t}-\alpha \frac{\partial}{\partial x}\left[(1+\sigma T) \frac{\partial T}{\partial x}\right]\right. \\
\left.-\alpha \frac{\partial}{\partial y}\left[(1+\sigma T) \frac{\partial T}{\partial y}\right]\right\}^{2} \mathrm{~d} x \mathrm{~d} y
\end{array}
$$

where $\left(x_{0}, y_{0}\right)$ and $\left(x_{1}, y_{1}\right)$ are to be selected in accordance with the contour in question. 
The minimization of $Z$ will be performed with respect to the temporal "complex" $\dot{\phi}$. Introducing (28) into (29), integrating, and omitting all terms not containing $\dot{\phi}$, we find:

$$
Z=I_{1} \dot{\phi}^{2}-2 \alpha I_{2} \phi \dot{\phi}-2 \alpha \sigma I_{3} \phi^{2} \dot{\phi}
$$

where

$$
\begin{aligned}
& I_{1}=\int_{x_{0}}^{x_{1}} \int_{y_{0}}^{y_{1}} B^{2}(x, y) \mathrm{d} x \mathrm{~d} y \\
& I_{2}=\int_{x_{0}}^{x_{1}} \int_{y_{0}}^{y_{1}} B(x, y)\left[\frac{\partial^{2} B}{\partial x^{2}}+\frac{\partial^{2} B}{\partial y^{2}}\right] \mathrm{d} x \mathrm{~d} y \\
& I_{3}=\int_{x_{0}}^{x_{1}} \int_{y_{0}}^{y_{1}}\left\{B^{2}(x, y)\left[\frac{\partial^{2} B}{\partial x^{2}}+\frac{\partial^{2} B}{\partial y^{2}}\right]\right. \\
& \left.+B\left[\left(\frac{\partial B}{\partial x}\right)^{2}+\left(\frac{\partial B}{\partial y}\right)^{2}\right]\right\} \mathrm{d} x \mathrm{~d} y .
\end{aligned}
$$

The condition

$$
\frac{\partial Z}{\partial \dot{\phi}}=0
$$

yields the differential equation

$$
I_{1} \dot{\phi}-I_{2} \alpha \phi-\alpha \sigma I_{3} \phi^{2}=0 .
$$

The initial condition $\phi(0)$ will be determined by minimizing the initial square residual of the form:

$$
\mathrm{J}=\int_{x_{0}}^{x_{1}} \int_{y_{0}}^{y_{1}}\left[T_{0}-\phi(0) B(x, y)\right]^{2} \mathrm{~d} x \mathrm{~d} y
$$

with respect to the arbitrary constant of the general solution of differential equation (32).*

As the particular examples we will consider two characteristic shapes of cross section.

(a) The triangle. Consider the triangular cross-section bounded by $x=0, y=0, x+y-l=0$. For this case the trial solution is supposed to be of the form:

$$
T=\phi(t)\left(1-\frac{x}{l}-\frac{y}{l}\right) \frac{x}{l} \frac{y}{l}
$$

hence

$$
B(x, y) \equiv\left(1-\frac{x}{l}-\frac{y}{l}\right) \frac{x}{l} \frac{y}{l}
$$

and the limits of integration in (31) are

$$
x_{0}=y_{0}=0, \quad x_{1}=l, \quad y_{1}=l-x .
$$

Integrating

$$
\dot{\phi}+\frac{56 \alpha}{l^{2}} \phi+\frac{\alpha \sigma}{l^{2}} \frac{2}{3} \phi^{2}=0
$$

we have

$$
\phi=\frac{-\exp \left(-56 \alpha t / l^{2}\right)}{\frac{\sigma}{84} \exp \left(-56 \alpha t / l^{2}\right)+\frac{C_{2} l^{2}}{56}}
$$

where $C_{2}$ is the constant of integration. The equation

$$
\frac{\partial J}{\partial C_{2}}=0
$$

* This standard procedure for finding the initial condition of $\phi(t)$ was applied by many authors previously. See for example [4]. will yield

$$
C_{2}=-\frac{2}{3 l^{2}} \sigma-\frac{56}{42 l^{2} T_{0}}
$$

hence the solution is of the form

$$
\begin{aligned}
T(x, y, t)=\frac{42 T_{0} \exp \left(-56 \alpha t / l^{2}\right)}{1+\sigma \frac{T_{0}}{2}\left[1-\exp \left(-56 \alpha t / l^{2}\right)\right]} \\
\times\left(1-\frac{x}{l}-\frac{y}{l}\right) \frac{x}{l} \frac{y}{l} .
\end{aligned}
$$

(b) Rectangular cross-section. Let the boundaries of a rectangle be defined by

$$
\begin{aligned}
& x= \pm l \\
& y= \pm b .
\end{aligned}
$$

For this case we will suppose

$$
B(x, y) \equiv\left(\frac{x}{l}-\frac{x^{2}}{l^{2}}\right)\left(\frac{y}{b}-\frac{y^{2}}{b^{2}}\right)
$$

and proceeding as in the previous case we find that the temperature distribution is

$$
T(x, y, t)=\frac{25 T_{0} \exp \left[-10 \frac{l^{2}+b^{2}}{l^{2} b^{2}} \alpha t\right]}{1+\frac{15}{28} \sigma T_{0}\left[1-\exp \left(-10 \frac{l^{2}+b^{2}}{l^{2} b^{2}} \alpha t\right)\right]}
$$

It is interesting to note that for the linear case $\sigma=0$, the corresponding results obtained from (38) and (41) are identical with those obtained by Tsoi in [5], who used the approximate method based on Laplace transforms. Tsoi reports that for the case of a rectangle his results are in good agreement with the exact solution. Unfortunately for more complicated geometry as triangular for example, the comparison is not possible because the exact analytical solution is not available.

It is reasonable to suppose that the solutions (38) and (41) are of some validity for the moderate range of parameter $\sigma$.

The corresponding results for both cases (a) and (b) are presented graphically on Figs. 1 and 2, where the influence of nonlinearity is presented also.

\section{(B) A melting problem}

Consider a semi-infinite solid initially at the melting temperature $\theta_{p}$ whose surface $x=0$ is raised suddenly to the temperature $\theta_{0}$ and held there for $t \geqslant 0$. We will assume the temperature distribution only in the liquid phase. Such a simplification was proposed by Goodman [6] and greatly enhances the use of penetration depth concept in trial solution as far as the latter becomes identical with the location of the melting line $\xi(t)$.

Introducing dimensionless temperature $T=\left(\theta-\theta_{p}\right) /$ $\left(\theta_{0}-\theta_{p}\right)$, and assuming a liquid of constant thermophysical properties, the governing equation is given by (13), where a modification is made introducing dimensionless time

$$
F o=\alpha t / l^{2}
$$




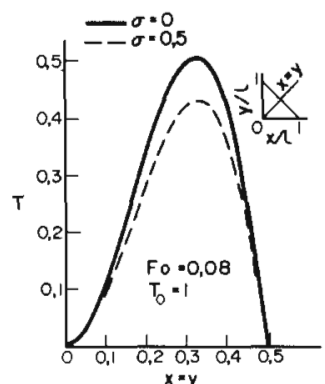

(a)

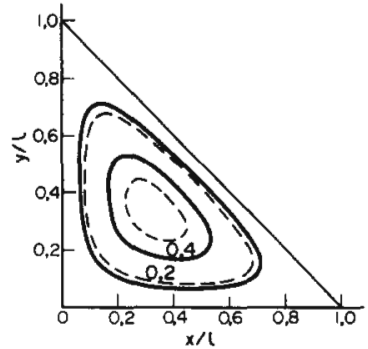

(b)
FIG. 1. Temperature distribution in the triangular crosssection at $F_{O}=\alpha t / 4 l^{2}=0.08$ for linear $(\sigma=0)$ and nonlinear $(\sigma=0.5)$ case, (a) temperature distribution in the center line $(x=y)$, (b) isothermals $T=0.2$ and 0.4 .

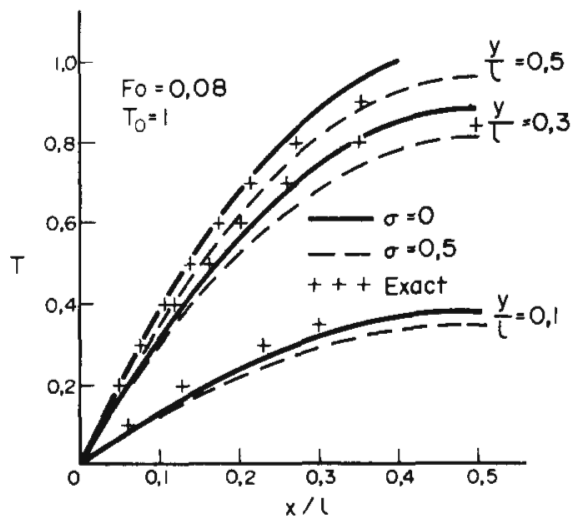

Frg. 2. Temperature distribution in the square cross-section at $F o=\alpha t / 4^{2}=0.08$ for linear $(\sigma=0)$ and nonlinear $(\sigma=0.5)$ case.

where $l$ is a characteristic length. The boundary condition at $x=0$ is

$$
T(0, F o)=1 \text { for } F o>0
$$

while the following two conditions have to be satisfied at the interface:

$$
\begin{gathered}
T(\xi, F o)=0 \\
-\frac{\partial T}{\partial X}=\frac{L}{c_{0}\left(\theta_{0}-\theta_{p}\right)} \frac{\mathrm{d} \xi}{\mathrm{d} F o} \text { for } X=\xi(F o) .
\end{gathered}
$$

In what follows, let $T(X, F o)$ be represented by a quadratic expression

$$
T=A\left(\frac{X}{\xi}-1\right)+A_{1}\left(\frac{X}{\xi}-1\right)^{2}
$$

which satisfies identically condition (44), and where the constants $A$ and $A_{1}$ are to be determined. It is evident, from (43), that $A_{1}=A+1$ so that profile (46) becomes

$$
T=A\left(\frac{X}{\xi}-1\right)+(A+1)\left(\frac{X}{\xi}-1\right)^{2}
$$

where only penetration depth $\xi(F o)$ and the constant $A$ remain for evaluation in accordance with the condition at the interface (45) and the governing heat-conduction equation (13).

Upon substituting (47) into equation (45), one is led to the following differential equation for $\xi$

$$
2 \xi \dot{\xi}=-A \mu
$$

where $\mu=2\left(\theta_{0}-\theta_{p}\right) c_{0} / L$, which when solved for the initial condition $\xi(0)=0$ gives

$$
\xi=2 \beta \sqrt{ }(F o)
$$

where

$$
\beta=\sqrt{ }(-A \mu / 4) \text {. }
$$

It can easily be seen that in this manner all aforementioned conditions are fulfilled by (47) and (49). But as trial function (47) fails to satisfy the governing differential equation (13), we may proceed, by forming the constraint (16). The temperature profile (47), when substituted into (16), and the integration with respect to $X$, this time from $X=0$ to $X=\xi(F o)$ performed, yields

$$
\begin{aligned}
Z=\frac{1}{15}\left(2 A^{2}-A\right. & +2) \frac{W^{2}}{\xi} \\
& +\frac{2}{3}\left(A^{2}-A-2\right) K W+4(A+1)^{2} K^{2} \xi
\end{aligned}
$$

where $W \equiv \dot{\xi}$ and $K \equiv 1 / \xi^{2}$ are physical components of $\partial T / \partial F O$ and $\partial^{2} T / \partial X^{2}$ respectively. We have two possibilities to obtain the approximate solution of the problem in consideration, i.e. by minimization of the constraint (51): (i) with respect to $W$, and (ii) with respect to $K$.

(i) Minimization with respect to spatial change of temperature $\mathrm{K}$. Condition $\partial Z / \partial K=0$ together with $K=1 / \xi^{2}$ yields the differential equation

$$
2 \xi \dot{\xi}=\frac{24(A+1)}{2-A} .
$$

Compatibility of this equation with equation (48) constitutes the approximate solution to the problem. From equations (52) and (48) we get algebraic equation

$$
\mu A^{2}-(2 \mu+24) A-24=0
$$

which determines $A$.

Since, according to (50) $A$ has to be less than zero we choose the negative root of quadratic equation (53) and obtain

$$
\beta=\frac{1}{2}\left[\left(\mu^{2}+48 \mu+144\right)^{\frac{1}{2}}-(\mu+12)\right]^{\frac{1}{2}}
$$

which completes the approximate solution.

(ii) Minimization with respect to temporal change of temperature $\mathbf{W}$. When borne in mind that $W=\dot{\xi}$, minimization of constraint (51) with respect to variations of $W,(\partial Z / \partial W=0)$ lead up to

$$
2 \xi \dot{\xi}=-10 \frac{A^{2}-A-2}{2 A^{2}-A+2} .
$$

Insisting on compatibility of equations (55) and (48) we obtain the following algebraic equation for $A$

$$
2 \mu A^{3}-(\mu+10) A^{2}+(2 \mu+10) A+20=0
$$

whose negative roots of relevance are plotted in Fig. 3 for $0 \leqslant \mu \leqslant 3$. (The interval considered for comparison with the exact solution.) Hence, in order to evaluate the approximate solution in this case, one has to use Fig. 3 and equations (49) and (50). It is worth noticing that the results just obtained in (55) and (56) are equivalent to those of the Galerkin method for the same trial function (47). 


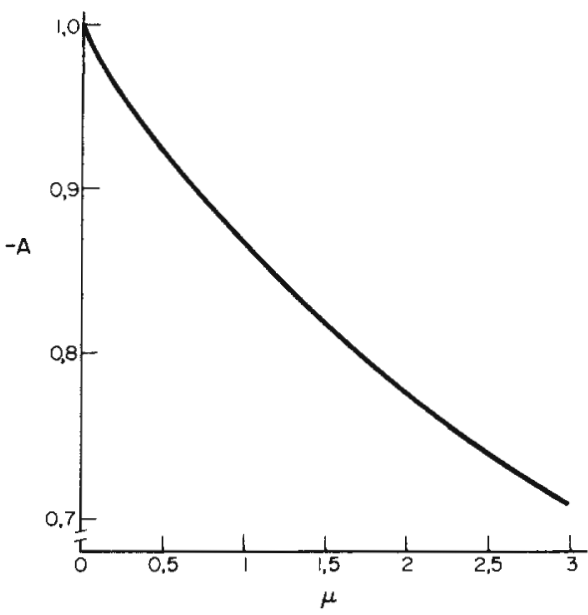

FIG. 3. Negative roots of equation (56).

In the exact solution $\mu$ is related to the melting constant $\beta$ by [7]:

$$
\mu=2(\pi)^{\frac{1}{2}} \beta \exp \left(\beta^{2}\right) \operatorname{erf}(\beta) .
$$

Since the relation $\beta=\beta(\mu)$ is of most interest in the solution of heat-conduction problems with moving boundaries, in Fig. 4 the percentage error $E=$ $100\left(\beta_{\text {approx }}-\beta_{\text {exact }}\right) / \beta_{\text {exact }}$ is plotted against the dimensionless quantity $\mu$ defined above. $\mu=3$ was the largest value of this parameter considered so that values required in practice are covered by this range.

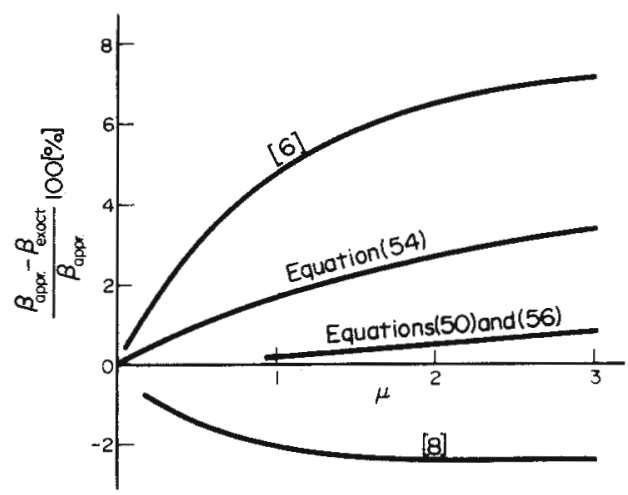

FIG. 4. Percentual error in determination of melting constant by different approximate methods.

The improvement of the accuracy for the approximate solutions obtained here, when compared with those obtained in [6] using the heat-balance integral, and in equation (5.28) of [8] by the help of a rather incorrect treatment of the problem, with a trial solution of the same form, is self-evident. We also conclude that the optimization with respect to temporal change of temperature field yields, in this case, much better results than the same with respect to spatial change of temperature.

(C) Semi-infinite body with an arbitrary heat flux input

As the last example, consider the case of the transient heat-conduction problem in the semi-infinite solid with constant thermal properties whose initial temperature is zero. In order to involve both linear and nonlinear boundary conditions the assumption will be made that the heat flux at the surface $x=0$ is an arbitrary function of surface temperature $T_{s}=T(0, t)$ and time. Hence, the problem can be stated mathematically as follows. The governing equation is (13), with initial condition

$$
T(x, 0)=0
$$

and boundary condition at $x=0$

$$
k_{0} \frac{\partial T}{\partial x}+f\left(T_{s}, t\right)=0
$$

Following references [6] and [9] let us assume the cubic temperature profile in the form:

$$
T=T_{s}(t)\left(1-\frac{x}{q(t)}\right)^{3}
$$

where $q=q(t)$ is the penetration depth and both $q$ and $T_{s}$ are unknown functions of time.

From the boundary condition (59) it follows that penetration depth and surface temperature are dependent

$$
3 k_{\mathrm{o}} T_{\mathrm{s}}=q f
$$

which indicates that we are going to deal with a constrained optimization problem in the appliance of the above exposed direct method to find $T_{s}$ and $q$. In the sense of this fact, we will use the Lagrange's multiplier technique, when dealing with the minimization with respect to physical components of temporal change of temperature.

Let us start by substituting the trial function, equation (60), into

$$
Z=\int_{0}^{q}\left[\left(\frac{\partial T}{\partial t}\right)^{2}-2 \alpha \frac{\partial T}{\partial t} \frac{\partial^{2} T}{\partial x^{2}}+\alpha^{2}\left(\frac{\partial^{2} T}{\partial x^{2}}\right)^{2}\right] \mathrm{d} x
$$

and performing the indicated integration, one obtains

$$
\begin{aligned}
Z=\frac{\dot{T}_{s}^{2} q}{7}+\frac{T_{s} \dot{T}_{s} \dot{q}}{7} & +\frac{3}{35} \frac{T_{s}^{2} \dot{q}^{2}}{q} \\
& -\frac{12}{5} \alpha \frac{T_{s} \dot{T}_{s}}{q}-\frac{9}{5} \alpha \frac{T_{s}^{2}}{q^{2}} \dot{q}+12 \alpha^{2} \frac{T_{s}^{2}}{q^{3}}
\end{aligned}
$$

where the dot represents differentiation with respect to time.

The next step is to recognize the physical components of temporal and spatial change of temperature in this expression so that the minimization procedures may be performed straightforwardly. Since

$$
\frac{\partial T}{\partial t}=\frac{\dot{T}_{s}}{q^{3}}(q-x)^{3}+\frac{3 T_{s}}{q^{4}}(q-x)^{2} x \dot{q}
$$

it is obvious that temporal change of temperature distribution possesses two natural components

$$
W_{1}=\dot{T}_{s} \quad \text { and } \quad W_{2}=\dot{q} .
$$

On the other hand spatial change of temperature

$$
\alpha \frac{\partial^{2} T}{\partial x^{2}}=6 \frac{T_{s}}{q^{2}}(q-x) \alpha
$$


has only one complex:

$$
K \equiv \alpha \frac{T_{s}}{q}
$$

In order to demonstrate two possible solutions we will solve the problem minimizing corresponding Gauss's constraint with respect to temporal and spatial change of the assumed temperature distribution respectively.

(i) Minimization with respect to temporal change. Omitting last term in (63) as irrelevant since it does not contain components $W_{1}$ and $W_{2}$ defined by (65), and using (61) whence

$$
3 k_{0} T_{s}-\dot{q} f-q\left(f^{\prime} T_{s}+f\right)=0
$$

where $f=\partial f / \partial t$ and $f^{\prime}=\partial f / \partial T_{s}$, we have

$$
\begin{aligned}
Z= & \frac{3 k_{0} T_{s} W_{1}^{2}}{7 f}+\frac{T_{s} W_{1} W_{2}}{7}+\frac{T_{s} f W_{2}^{2}}{35 k_{0}} \\
& -\frac{4}{5} \frac{\alpha f}{k_{0}} W_{1}-\frac{1}{5} \frac{\alpha f^{2}}{k_{0}^{2}} W_{2} \\
& +\lambda\left(3 k_{0} W_{1}-f W_{2}-\frac{3 k_{0} T_{s} W_{1} f^{\prime}}{f}-\frac{3 k_{0} T_{s} f}{f}\right)
\end{aligned}
$$

where $\lambda$ is an unknown Lagrange's multiplier.

Minimization with respect to $\partial T / \partial t$, i.e.

$$
\frac{\partial Z}{\partial W_{1}}=0 \text { and } \frac{\partial Z}{\partial W_{2}}=0
$$

yields the following system of differential equations $30 k_{0}^{2} T_{s} T_{s}+5 f k_{0} T_{s} \dot{q}-28 \alpha f^{2}$

$$
\begin{array}{r}
+105 \lambda k_{0}^{2} f\left(1-\frac{T_{s} f^{\prime}}{f}\right)=0 \\
5 k_{0}^{2} T_{s} \dot{T}_{s}+2 f k_{0} T_{s} \dot{q}-7 \alpha f^{2}-35 \lambda k_{0}^{2} f=0 .
\end{array}
$$

or

$$
T_{s}=\frac{f}{k_{0}}\left(\frac{49}{39} \alpha t\right)^{\frac{t}{t}}=1.121 \frac{f}{k_{0}}(\alpha t)^{\frac{t}{t}}
$$

2. If $f=f(t)$, equation (74) becomes

$$
2 T_{s} \dot{T}_{s}+\frac{11}{13} \frac{T_{s}^{2} f}{f}=\frac{49}{39} \frac{\alpha f^{2}}{k_{0}^{2}}
$$

and

3. When $f=f\left(T_{s}\right)$, equation (74) yields

$$
\begin{aligned}
2 T_{s} \dot{T}_{s}-2 \frac{T_{s}^{2} \dot{T}_{s}}{f} f^{\prime}+{ }_{13}^{6} \frac{T_{s}^{3} \dot{T}_{s} f^{\prime 2}}{f^{2}} & \\
& =\frac{49}{39} \frac{\alpha f^{2}}{k_{0}^{2}}-\frac{7}{13} \frac{\alpha f f^{\prime} T_{s}}{k_{0}^{2}} .
\end{aligned}
$$

Assuming that, in second case, $f=f_{0} \cdot t^{N}$ where $N$ is an integer, equation (77) becomes

$$
t\left(T_{s}\right)^{2}+\frac{14}{13} N T_{s}^{2}=\frac{49}{3 f_{0}^{2}} t^{2 N+1}
$$

and reduces to the first case for $N=0$. The solution to equation (79) is

$$
T_{s}=\frac{f_{0}}{k_{0}}\left(\frac{49 \alpha t}{45 N+39}\right)^{t} t^{N}
$$

Upon separation of variables the differential equation for the third case (78) yields

$$
\int_{0}^{T_{s}} \frac{78 T_{s} f^{2}-66 T_{s}^{2} f f^{\prime}+18 T_{s}^{3} f^{\prime 2}}{f^{3}\left(7 f-3 T_{s} f^{\prime}\right)} \mathrm{d} T_{s}=\frac{7 \alpha t}{k_{0}^{2}} .
$$

Let us solve this equation for the case of power low heat transfer at the body surface into a medium at zero temperature, i.e.

$$
f\left(T_{s}\right)=H\left(T_{s}-T_{0}\right)^{m}
$$

where $H$ and $m$ are constants. Introducing $\eta=1+T_{s} / T_{0}$ equation (81) becomes

$$
\int_{1}^{\eta} \frac{\left(13-11 m+3 m^{2}\right) \eta^{3}-\left(13-22 m+9 m^{2}\right) \eta^{2}+(9 m-11) m \eta-3 m^{2}}{\eta^{2 m+1}[3 m-(3 m-7) \eta]} \mathrm{d} \eta=\frac{7}{6} \frac{T_{0}^{2 m-2} H^{2}}{k_{0}^{2}} \alpha t .
$$

Upon elimination $\lambda$ from equations (71) and (72) one is led to

$$
\begin{aligned}
45 k_{0}^{2} T_{s} \dot{T}_{s}+11 f k_{0} T_{s} \dot{q}-49 \alpha f^{2}-15 k_{0}^{2} T_{s}^{2} \dot{T}_{s} \frac{f^{\prime}}{f} \\
-6 k_{0} T_{s}^{2} \dot{q} f^{\prime}+21 \alpha f T_{s} f^{\prime}=0
\end{aligned}
$$

which when combined with equations (68) and (61) gives the first order differential equation for the temperature at the surface

$$
\begin{aligned}
2 T_{s} \dot{T}_{s}-\frac{22}{13} \frac{T_{s}^{2}}{f}\left[\left(1-\frac{3}{11} \frac{T_{s} f^{\prime}}{f}\right)\right. & \left.\left(f^{\prime} \dot{T}_{s}+f\right)-\frac{1}{2} f\right] \\
& =\frac{49}{39} \frac{\alpha f^{2}}{k_{0}^{2}}-\frac{7}{13} \frac{\alpha f f^{\prime} T_{s}}{k_{0}^{2}} .
\end{aligned}
$$

The initial condition for the problem is $T_{s}(0)=0$. There are three cases in which equation (74) can be integrated analytically:

1. $f=$ const. In this case equation (74) reduces to

$$
2 T_{s} \dot{T}_{s}=\frac{49}{39} \frac{\alpha f^{2}}{k_{0}^{2}}
$$

The method of handling (82) applies also to the case in which the surface flux is a sum of powers of the surface and environment temperatures, and thus to heat convection from bounding surface into a fluid at temperature $T_{f}$, for which the boundary condition is (linear case) $f=h\left(T_{s}-T_{f}\right)$, and also to the blackbody radiation into a medium at temperature $T_{m}: f=\sigma\left(T_{s}^{4}-T_{m}^{4}\right)$. Final results obtained from (83) are:

(a) for $m=4 ; H=\sigma$

$$
\frac{T_{0}^{6} \sigma^{2}}{k_{0}^{2}} \alpha t=\frac{1}{\eta^{8}} \sum_{i=0}^{7} a_{i} \eta^{i}+b \ln \frac{12-5 \eta}{7 \eta}+c
$$

where

$$
\begin{array}{llrl}
a_{0} & =3 / 7 & a_{5} & =-275 / 31104 \\
a_{1} & =-40 / 49 & a_{6} & =-1375 / 248832 \\
a_{2} & =107 / 252 & a_{7} & =-6875 / 1492992 \\
a_{3} & =-11 / 360 & b & =-34375 / 17915904 \\
a_{4} & =-55 / 3456 & c & =376528644 / 13168189440
\end{array}
$$


(b) for $m=2$

$$
\begin{aligned}
\frac{T_{0}^{2} H^{2}}{k_{0}^{2}} \alpha t=\frac{1}{\eta^{4}}\left[\frac{3}{4}-\frac{16}{21} \eta+\frac{23}{42} \eta^{2}\right. & \left.-\frac{11}{18} \eta^{3}\right] \\
& +\frac{11}{108} \ln \frac{6+\eta}{7 \eta}+\frac{25}{63} .
\end{aligned}
$$

In linear case $(m=1)$ the result is

$$
\frac{h^{2}}{k_{0}^{2}} \alpha t=\frac{3 \eta^{2}-12 \eta+9}{21 \eta^{2}}+\frac{77}{42} \ln \frac{4 \eta+3}{7}-\frac{16}{21} \ln \eta
$$

where $\eta=1-T_{s} / T_{f}$ since the boundary condition is taken of the form $f=h\left(T_{f}-T_{s}\right)$.

The discussion of the results given above will take place after solving the same problems using the second possibility, viz. minimizing the constraint (63) with respect to the component of spatial change of temperature.

(ii) Minimization with respect to spatial change. This time omission of terms not containing component $K$ defined by (67) reduces constraint (63) to the following expression of relevance:

$$
Z=-\frac{12}{5} K T_{s}-\frac{2}{5} K \frac{T_{s}}{q} \dot{q}+12 \frac{K^{2}}{q} .
$$

Optimization condition

$$
\frac{\partial Z}{\partial K}=0
$$

together with (67) will yield, upon using (61) and (68), the differential equation

$$
63 T_{s} \dot{T}_{s}-27 \frac{T_{s}^{2}}{f}\left(T_{s} f^{\prime}+f\right)=40 \frac{\alpha f^{2}}{k_{0}^{2}}
$$

determining surface temperature.

If we consider, as above, the cases for which equation (89) is integrable, the following reductions are evident:

1. $f=$ const.

with the solution

$$
2 T_{s} T_{s}=\frac{80}{63} \frac{\alpha f^{2}}{k_{0}^{2}}
$$

$$
T_{s}=1.127 \frac{f}{k_{0}}(\alpha t)^{\frac{1}{2}}
$$

2. $f=f(t)$

$$
2 T_{s} \dot{T}_{s}-\frac{6}{9} \frac{T_{s}^{2} f}{f}=\frac{\mathrm{g} g}{6} \frac{a f^{2}}{k_{0}^{2}}
$$

3. $f=f\left(T_{s}\right)$

$$
\text { ㅇํ의 } \int_{0}^{T_{s}} \frac{14 T_{s} f-6 T_{2}^{2} f^{\prime}}{f^{3}} \mathrm{~d} T_{s}=\frac{\alpha t}{k_{0}^{2}} .
$$

As previously in the second case we choose $f=f_{0} \cdot t^{N}$ to get the solution of (92)

$$
T_{s}=\frac{f_{0}}{k_{0}}\left(\frac{80 \alpha t}{72 N+63}\right)^{t} t^{N}
$$

Assuming again $f\left(T_{s}\right)=T_{0}^{m} \cdot H \cdot \eta^{m}$ where $\eta=1+T_{s} / T_{0}$ we obtain the solution for the third case in the form

$\frac{9}{40} \int_{1}^{\eta} \frac{(7-3 m) \eta^{2}+(6 m-7) \eta-3 m}{\eta^{2 m+1}} \mathrm{~d} \eta=\frac{T_{0}^{2 m-2} H^{2}}{k_{0}^{2}} \alpha . t$ (95) which can very easily be integrated for particular values of $m$. Thus,

(a) for $m=4 ; H=\sigma$

$$
\frac{T_{0}^{6} \sigma^{2}}{k_{0}^{2}} \alpha t=\frac{1}{\eta^{8}}\left(\frac{27}{80}-\frac{153}{280} \eta+\frac{3}{16} \eta^{2}\right)+\frac{3}{140}
$$

(b) for $m=2$

$$
\frac{T_{0}^{2} H^{2}}{k_{0}^{2}} \alpha t=\frac{1}{\eta^{4}}\left(\frac{27}{80}-\frac{3}{8} \eta-\frac{9}{8} \eta^{2}\right)+\frac{3}{20}
$$

(c) for $m=5 / 4$

$$
\frac{T_{0}^{1 / 2} H^{2}}{k_{0}^{2}} \alpha t=\frac{1}{\eta^{5 / 2}}\left(\frac{27}{80}-\frac{3}{40} \eta-\frac{117}{80} \eta^{2}\right)+\frac{6}{5} .
$$

Finally in the case of heat convection boundary condition $f\left(T_{s}\right)=h\left(T_{f}-T_{s}\right)$ from equation (93) one obtains

$$
\frac{h^{2}}{k_{0}^{2}} \alpha t=\frac{9}{10}\left(\ln \eta+\frac{1}{4 \eta}+\frac{3}{8 \eta^{2}}\right)-\frac{9}{16}
$$

where $\eta=1-T_{s} / T_{f}$.

Let us now proceed with the discussion and the comparison of the results. One thing is obvious-the closed form solutions obtained by minimization of Gauss's constraint with respect to the component of spatial change of temperature distribution are more attractive since they are of a rather less complicated form. To evaluate the accuracy of the results we are going to compare all of them with the exact solutions for surface temperature.

In the case of constant heat flux the exact solution is known to be $T_{s}=1.128\left(f / k_{0}\right)(\alpha t)^{t}$, which when compared with equations (76) and (91) indicates the

\begin{tabular}{|c|c|c|c|c|}
\hline \multirow[b]{2}{*}{$N$} & \multicolumn{4}{|c|}{ Ratios $r(N)$, equation (100), corresponding to: } \\
\hline & $\begin{array}{c}\text { Equation } \\
(80)\end{array}$ & $\begin{array}{c}\text { Equation } \\
(94)\end{array}$ & [6] & {$[9]$} \\
\hline 0 & 0.993 & 0.999 & 1.023 & 1.000 \\
\hline 1 & 1.015 & 1.023 & 1.085 & 1.085 \\
\hline 2 & 1.024 & 1.033 & 1.108 & 1.117 \\
\hline 3 & 1.029 & 1.038 & 1.119 & 1.133 \\
\hline 4 & 1.032 & 1.041 & 1.126 & 1.143 \\
\hline 5 & 1.033 & 1.043 & 1.131 & 1.149 \\
\hline 6 & 1.035 & 1.045 & 1.134 & 1.154 \\
\hline 8 & 1.037 & 1.047 & 1.139 & 1.160 \\
\hline 10 & 1.038 & 1.048 & 1.141 & 1.164 \\
\hline 12 & 1.073 & 1.049 & 1.144 & 1.168 \\
\hline
\end{tabular}
improvement of the result obtained by the help of minimization with respect to $K$. But when compared with the result $1.15\left(f / k_{0}\right)(\alpha t)^{\frac{1}{2}}$ of application of the integral method [6] to the same problem, both results are better.

To compare the results for the case when heat flux is the power function of time $\left(f=f_{0} \cdot t^{N}\right)$ we calculate

$$
r(N)=\frac{T_{\text {sapprox. }}}{T_{\text {sexact }}}=\frac{\Gamma\left(N+\frac{3}{2}\right)}{\Gamma(N+1)} \cdot R(N)
$$

where $R(N)=[49 /(45 N+39)]^{1 / 2}$ for the approximate solution $(80)$ or $R(N)=[80 /(72 N+63)]^{1 / 2}$ if $(94)$ is considered. The ratios $(100)$ are given in Table 1 for the values of power $N$ from 0 to 12 . In the same table

Table 1 
the corresponding ratios for the approximate solutions obtained by variational [9] and integral [6] method are also listed.

Comparison of the approximate solutions with the numerical ones [10], for the case when the heat flux is a power low function of the surface temperature, is evident from Fig. 5. The dots represent approximation equations (96), (97) and (98) for $m=4,2$ and $5 / 4$ respectively. If one superimposes the results of Goodman [6], or Vujanovic and Strauss [9] (available only for $m=4$ ), or those obtained by minimization with respect to $\partial T / \partial t$ in this paper, it may be concluded that all of them are less accurate.

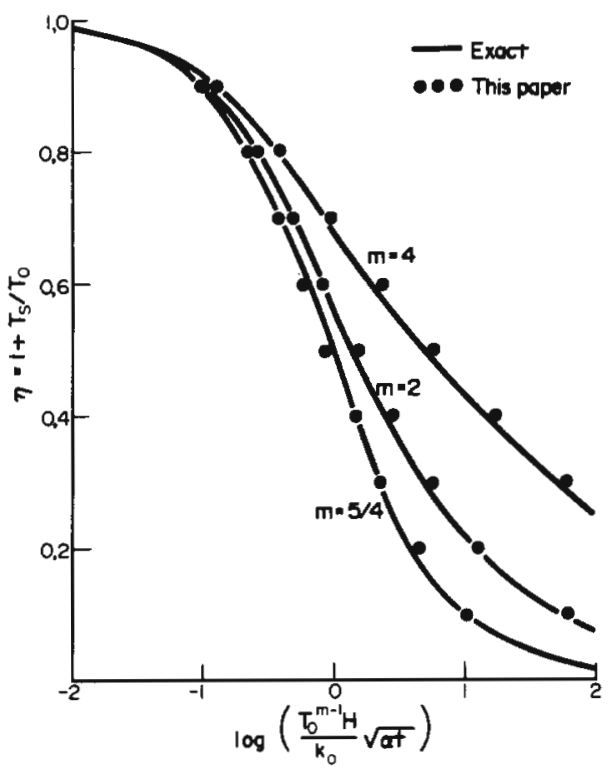

FIG. 5. Surface temperature of the semi-infinite solid with surface flux the $m$ th power of the temperature. The dots represent approximation equations (96), (97) and (98).

When the solution of equation (99) is compared with the exact one, and the percentage error

$$
E=100\left(\eta_{\text {approx. }}-\eta_{\text {exact }}\right) / \eta_{\text {exact }}
$$

is calculated it turns out that $E=3 \%$, for $\left(T_{s} / T_{f}\right)_{\text {approx. }}=$ 0.8 , which reduces to $1 \%$ for $\left(T_{s} / T_{s}\right)_{\text {approx. }}=0.5$, while for $\left(T_{s} / T_{f}\right)_{\text {approx. }} \leqslant 0.3, E \leqslant 1 \%$. Doing the same with equation (86): $E=6.4 \%$ at $\left(T_{s} / T_{f}\right)_{\text {approx. }}=0.8 ; E=1 \%$ at $\left(T_{s} / T_{f}\right)_{\text {approx. }}=0.4$ and less than $0.5 \%$ for $\left(T_{s} / T_{f}\right)_{\text {approx. }}<0.3$.

Thus, in all cases the solutions here obtained differ only slightly from the exact ones. Further, the important conclusion is that the result obtained by minimization of Gauss's constraint with respect to the component of spatial change of temperature distribution are always more accurate, although of rather less complicated form.

\section{DISCUSSION}

In conclusion several remarks would be of interest:

1. The method we have presented is quite useful in the search for approximate solutions of linear and nonlinear heat-conduction problems. Usually, in the case of nonlinear analysis, it pertains to a high accuracy when compared with the other approximate methods.

2. A common feature of all approximate methods in heat transfer, including the method presented, is that the solution of a problem should be selected to some extent a priori. The choice of the form of a solution which contains some parameters that should be determined by the help of the Gauss's method depends upon all the information available from empirical, experimental, intuitive, etc. data.

3. Our aim has been to demonstrate two possibilities for obtaining approximate sólutions from the same Gauss's constraint. The question as to which of the two possible approximate solutions should be taken is to be decided by considering the mean square residual [11] (p. 388) in the form

$$
Z=\int_{t_{0}}^{t_{1}} \int_{V}(X-Y)^{2} \mathrm{~d} V \mathrm{~d} t
$$

Naturally, the value of $Z$ defined by (101) is equal to zero for the exact solution. Hence, the better solution is that one for which $Z$ has the smallest value. It should be pointed out that the same criteria can be employed for evaluating the accuracy of any approximate solution obtained by some other approximate method.

4. The extension of the method presented in this paper on some more elaborated mathematical schemes as for example the method of finite elements is possible and will be reported elsewhere.

5. This method can be extended also in a straightforward way to handle the numerous problems arising in transport phenomena.

\section{REFERENCES}

1. W. Heisenberg, Philosophic Problems of Nuclear Sciences. Premier Book, New York (1966).

2. B. Vujanovic, The practical use of Gauss's principle of least constraint, J. Appl. Mech. to be published.

3. Yu. A. Samoilovich, Gauss's principle in heat conduction theory, Teplofiz. Vysokh. Temper. 12, 354-358 (1974).

4. L. Collatz, The Numerical Treatment of Differential Equations. Springer, Berlin (1960).

5. P. V. Tsoi and U. H. Kamarov, Transient heat conduction in the cylindrical bodies of arbitrary cross-section, in Research in Transient Heat and Mass Transfer, edited by A. V. Luikov and B. M. Smoljski. Nauka i Tehnika, Minsk (1966) (in Russian).

6. T. R. Goodman, Application of integral methods to transient nonlinear heat transfer, in Advances in Heat Transfer, edited by $\mathrm{T}$. Irvine and J. Hartnett. Academic Press, New York (1964).

7. H. S. Carslaw and J. C. Jaeger, Conduction of Heat in Solids, 2nd edn. Oxford University Press, Oxford (1959).

8. E. R. G. Eckert and R. H. Drake, Analysis of Heat and Mass Transfer. McGraw-Hill, New York (1972).

9. B. Vujanovic and A. M. Strauss, Heat transfer with nonlinear boundary conditions via a variational principle, $A I A A J / 9,327-330$ (1971).

10. J. C. Jaeger, Conduction of heat in a solid with a power law of heat transfer at its surface, Proc. Camb. Phil. Soc. 20, 634-641 (1950).

11. B, A. Finlayson, The Method of Weighted Residuals and Variational Principles. Academic Press, New York (1972). 


\section{APPLICATION DU PRINCIPE DE MOINDRE CONTRAINTE DE GAUSS AU PROBLEME DU TRANSFERT THERMIQUE NON-LINEAIRE}

Résumé-On présente une méthode approchée directe de résolution des problèmes de conduction thermique linéaires et non-linéaires, basée sur le principe de moindre contrainte de Gauss. Dans chaque cas particulier, le problème se réduit à la minimisation algébrique d'une forme quadratique par rapport à un ensemble de paramètres physiques. Quelques exemples concrets mettent en évidence l'efficacité et la précision de cette nouvelle méthode.

\section{DIE ANWENDUNG DES GAUSS'SCHEN PRINZIPS DES KLEINSTEN ZWANGES AUF DIE NICHTLINEAREN WÄRMEÜBERTRAGUNGSPROBLEME}

Zusammenfassung-Es wird eine auf dem Gauß 3 'schen Prinzip des kleinsten Zwanges gegründete, direkte Näherungsmethode für die Lösung linearer und nichtlinearer Wärmeleitungs probleme dargestellt. In jedem einzelnen Fall wird das Problem auf die algebraische Minimisation der quadratischen Form in Bezug auf einen bestimmten Komplex der physikalischen Parameter zurückgeführt. Anhand einiger konkreter Beispiele wird die Wirksamkeit und Genauigkeit dieser Methode dargelegt.

\section{ПРИМЕНЕНИЕ ПРИНЦИПА НАИМЕНЫШЕГО ПРИНУЖДЕНИЯ ГАУССА К ПРОБЛЕМЕ НЕЛИНЕЙНОГО ПЕРЕНОСА ТЕПЛА}

Аннотация - В работе представлен прямой метод приближенного решения линейных и нелинейных задач теплопроводности, основанный на принципе наименьшего принуждения Гаусса. В каждом частном случае проблема сводится к минимизации квадратичной формы по некоторому комплексу физических параметров. На нескольких конкретных примерах показана эффективность и точность нового метода. 